\title{
An effectiveness-implementation hybrid type 1 trial assessing the impact of group versus individual antenatal care on maternal and infant outcomes in Malawi
}

Ellen Chirwa ${ }^{1}$, Esnath Kapito ${ }^{1}$, Diana L. Jere ${ }^{1}$, Ursula Kafulafula ${ }^{1}$, Elizabeth Chodzaza ${ }^{1}$, Genesis Chorwe-Sungani ${ }^{1}$, Ashley Gresh², Li Liư ${ }^{3}$, Elizabeth T. Abrams', Carrie S. Klima4, Linda L. McCreary ${ }^{4}$, Kathleen F. Norr ${ }^{4}$ and Crystal L. Patil ${ }^{4^{*}}$ (D)

\begin{abstract}
Background: Sub-Saharan Africa has the world's highest rates of maternal and perinatal mortality and accounts for two-thirds of new HIV infections and $25 \%$ of preterm births. Antenatal care, as the entry point into the health system for many women, offers an opportunity to provide life-saving monitoring, health promotion, and health system linkages. Change is urgently needed, because potential benefits of antenatal care are not realized when pregnant women experience long wait times and short visits with inconsistent provisioning of essential services and minimal health promotion, especially for HIV prevention. This study answers WHO's call for the rigorous study of group antenatal care as a transformative model that provides a positive pregnancy experience and improves outcomes.
\end{abstract}

Methods: Using a hybrid type 1 effectiveness-implementation design, we test the effectiveness of group antenatal care by comparing it to individual care across 6 clinics in Blantyre District, Malawi. Our first aim is to evaluate the effectiveness of group antenatal care through 6 months postpartum. We hypothesize that women in group care and their infants will have less morbidity and mortality and more positive HIV prevention outcomes. We will test hypotheses using multi-level hierarchical models using data from repeated surveys (four time points) and health records. Guided by the consolidated framework for implementation research, our second aim is to identify contextual factors related to clinic-level degree of implementation success. Analyses use within and across-case matrices.

Discussion: This high-impact study addresses three global health priorities, including maternal and infant mortality, HIV prevention, and improved quality of antenatal care. Results will provide rigorous evidence documenting the effectiveness and scalability of group antenatal care. If results are negative, governments will avoid spending on less effective care. If our study shows positive health impacts in Malawi, the results will provide strong evidence and valuable lessons learned for widespread scale-up in other low-resource settings. Positive maternal, neonatal, and HIV-related outcomes will save lives, impact the quality of antenatal care, and influence health policy as governments make decisions about whether to adopt this innovative healthcare model.

Trial registration: ClinicalTrials.gov registration number NCT03673709. Registered on September 17, 2018.

Keywords: Antenatal care, Group healthcare, Maternal and newborn health, Preterm birth, Implementation, Fidelity, CFIR, Sub-Saharan Africa

\footnotetext{
*Correspondence: cpatil@uic.edu

${ }^{4}$ University of Illinois at Chicago, College of Nursing, 845 S. Damen Avenue

(M/C 806), Chicago, IL 60612, USA

Full list of author information is available at the end of the article
}

(c) The Author(s). 2020 Open Access This article is distributed under the terms of the Creative Commons Attribution 4.0 International License (http://creativecommons.org/licenses/by/4.0/), which permits unrestricted use, distribution, and reproduction in any medium, provided you give appropriate credit to the original author(s) and the source, provide a link to the Creative Commons license, and indicate if changes were made. The Creative Commons Public Domain Dedication waiver (http://creativecommons.org/publicdomain/zero/1.0/) applies to the data made available in this article, unless otherwise stated. 


\section{Background}

Sub-Saharan Africa has the world's highest maternal mortality ratio and a large proportion of people living with HIV [1]. Obstetric hemorrhage and hypertensive disorders, aggravated by HIV, account for many maternal deaths [2,3]. There have been substantial declines in under-five mortality, but neonatal mortality remains a public health challenge. Preterm birth is the leading cause of neonatal death $[4,5]$. Further, surviving premature infants suffer due to higher risk for later mortality and morbidity, neurodevelopmental impairment, developmental delays, and stunting [4-6].

Antenatal care provides an opportunity for life-saving monitoring, health promotion, and health system linkages through early detection and timely intervention [79]. In sub-Saharan Africa, however, provider shortages, resource stockouts, and disrespectful care translate into long wait times and short antenatal care visits at which there is inconsistent provisioning of essential services and health promotion [10-12]. Providers' failure to deliver essential services along with low attendance after the intake visit combine to reduce opportunities for early detection and timely intervention [13, 14]. For example, pre-eclampsia, a leading cause of maternal and perinatal death, can be addressed with reliable detection through blood pressure monitoring and early recognition of dangers signs, but less than half of women have their blood pressure measured at each antenatal care visit $[15,16]$. Health promotion is minimal, especially for HIV prevention, so exposure to HIV from untested partners is still a problem. Gaps persist in the continuum of HIV care for HIV-infected women and their infants during and after pregnancy $[13,14,17]$. When women can, they choose health facilities they perceive as having better quality of care because their healthcare experiences drive their care-seeking behavior; greater satisfaction is associated with attending more antenatal care visits [18-23]. Change is urgently needed to optimize the impact of antenatal care in sub-Saharan Africa.

To address antenatal care quality gaps, WHO recently revised their recommendations and doubled the number of contacts from 4 to 8 and to emphasize a positive pregnancy experience [22, 24]. How will low-resource health systems simultaneously double the number of visits and improve quality? A major paradigm shift in the current individual antenatal care model is needed. A promising model WHO identified as needing further exploration in the context of rigorous research is group antenatal care [24]. In group antenatal care, 8-12 women with similar gestational ages attend all of their visits together and see the same midwife over the course of the pregnancy.

CenteringPregnancy® is the only group antenatal care model with a large body of rigorous evidence supporting its effectiveness and the feasibility of bringing it to scale [25-27]. A two hour CenteringPregnancy group antenatal care visit includes self and midwife health assessments in a group space, interactive learning, and community building. Women measure their own blood pressure and weight, briefly consult the midwife in a corner of the room, and then meet for 80-90 min of interactive health promotion discussion enlivened by activities, games, and role-plays. Women form relationships with providers and one another as they collaboratively generate strategies to improve health across the pregnancy and into the early postpartum [25].

US-based randomized trials and a matched cohort study implemented with high fidelity to the model showed significant declines in prematurity rates and improved attendance, satisfaction, breastfeeding practices, safer sex behaviors, and uptake of family planning [2630]. In one randomized control trial (RCT), higher levels of women's engagement in interactive discussions related to a greater reduction of risk for prematurity [31]. When HIV and STI prevention was integrated into CP (termed $\mathrm{CP}+$ ), women had increased condom use and fewer repeat pregnancies [28]. This body of research shows the power that the CenteringPregnancy model has to improve antenatal care. Group antenatal care fundamentally alters service delivery, allows for longer and woman-centered care, and has the potential to meet demands for higher quality care.

To expand the benefits of group antenatal care to subSaharan Africa, we adapted the only evidence-based group antenatal care model, CenteringPregnancy, for use in Malawi and Tanzania [32-36]. To ensure that the model was adapted with fidelity to CenteringPregnancy's core components and associated practices and would be replicable, we consulted with the model's developer, Sharon Rising. We then conducted a 2-arm randomized pilot in which pregnant women $(n=218)$ were randomly assigned to individual (usual) focused antenatal care or group antenatal care (intervention). Significantly more women in group than individual antenatal care completed $\geq 4$ visits ( $94 \%$ vs $58 \%$ ) and attended a postnatal visit ( $75 \%$ vs $50 \%)$. Rates of partner HIV testing were higher for those in group antenatal care $(51 \%$ vs. $27 \%)$. Other positive outcomes included higher satisfaction, more HIV-related knowledge, and less mental distress. The showed that that group antenatal care can be offered with fidelity and that individual randomization was feasible and acceptable, supporting the need for a rigorous effectiveness trial [33].

In addition to our work, research conducted in Ghana [37] and Iran [38] were cited by the WHO to support group antenatal care's potential for impact [24]. However, the WHO was cautious, because premature adoption of a new model can have serious negative 
consequences, as exemplified by the reversal of the 4visit model [39]. A growing body of literature shows that group antenatal care is feasible and acceptable in many low and middle-income countries across the globe [4050]. Additional work is underway in Mali, China, Ethiopia, Surinam, and elsewhere [51]. Most of these studies do not yet have results, but 3 studies in Africa have noted positive outcomes [43, 52, 53]. A large cluster-randomized trial in Nigeria and Kenya found greater antenatal care attendance in both countries and more birth planning for a facility-based birth in Nigeria $[52,53]$. Two smaller studies in Nigeria with weaker designs reported greater attendance, greater knowledge of danger signs, and more use of health facilities for delivery $[54,55]$. Importantly, these initiatives do not report sufficient detail about if and in what ways their models deviate from the evidence-based CenteringPregnancy model (i.e., fidelity). It is not clear if the evidence-base practices associated with the positive outcomes of CenteringPregnancy were retained in these adaptations [25]. Additionally, many studies lack adequate power to test for effects on birth outcomes (e.g., prematurity and/or low birthweight). One large cluster randomized trial currently underway in Rwanda is adequately powered to examine preterm birth, but the model deviates on three key practices of the CenteringPregnancy model (e.g., lack of continuity of co-facilitators and group members (women can drop into other groups) and group size can be larger than 12) [42, 43]. Our study, taking place in Malawi, fills both the power and fidelity gaps while implementing group antenatal care in the context of a midwife shortage and high rates of prematurity and HIV infection [13, 56, 57].

\section{Methods}

\section{Study aims}

Aim 1 (effectiveness)

Using a randomized controlled trial (RCT) with individual randomization, we evaluate the effectiveness of group antenatal care through 6 months postpartum. We hypothesize that compared to individual care, women in group care and their infants will have less morbidity and mortality and more positive HIV prevention outcomes, including:

H1. Fewer preterm births (primary outcome), stillbirths, low birthweight infants and neonatal and maternal mortality.

H2. Higher rates of HIV testing for partners (primary outcome) and women at first visit and in late pregnancy if HIV negative, and more HIV prevention knowledge and behaviors.

H3. More optimal secondary outcomes: healthcare utilization, satisfaction with care, pregnancy-related knowledge, healthy behaviors, mental distress, anemia, hypertension, postpartum bleeding ( $>500 \mathrm{ml})$, exclusive breastfeeding, uptake of family planning and early repeat pregnancy.

H4. Exploratory: For the subset of $\sim 130 \mathrm{HIV}$-infected women: received antiretroviral therapy (ART) from intake through 6 months postpartum and infant HIV status known.

\section{Aim 2 (implementation)}

We identify clinic-level degree of implementation success and contextual factors associated with success for each clinic and across clinics.

\section{Design}

This study uses a hybrid type 1 design [58], to evaluate effectiveness and document implementation processes at six clinics in Blantyre District, Malawi.

\section{Study setting}

The Malawi Ministry of Health is preparing to adapt and rollout the WHO model nationwide [24]. Although this 8-visit model is not yet the national standard of care, the Ministry of Health felt that to be equitable, our RCT should offer eight antenatal care contacts to women in both group and individual study conditions. To enhance generalizability, we selected rural, periurban, and urban clinics that maximized variability in client volume (the key factor affecting implementation in our pilot) and staffing in Blantyre District, Malawi (Table 1). The variability in these clinics will allow us to assess if government-run clinics can implement group antenatal care across the spectrum of everyday clinic conditions. We also ensure that each clinic has the same equipment available to both the control and intervention study conditions.

\section{Aim 1 Study population (effectiveness)}

The study population is comprised of pregnant women over age 14 and less than 24 weeks pregnant. Those under age 15 , more than 24 weeks pregnant, or unable to make an informed choice (e.g. unable to converse about the study) are ineligible by design.

However, during the ethical approval process, our design had to be modified because of the recent change to

Table 1 Clinic staffing and volume

\begin{tabular}{lll}
\hline Clinic & Number of Midwives & New clients per month \\
\hline 1 & 3 & $40-50$ \\
2 & 7 & $80-90$ \\
3 & 15 & $50-60$ \\
4 & 11 & $80-90$ \\
5 & 12 & $85-90$ \\
6 & 16 & $200-250$ \\
\hline
\end{tabular}


the law forbidding marriage for women under the age of 18. At present, enrollment of minors raises the issue of needing to report the marriage as criminal. The US practice of issuing a certificate of confidentiality to avoid exposing research participants to legal jeopardy is not possible in Malawi. Until this issue is resolved the University of Malawi College of Medicine Research and Ethics Committee is not approving research with pregnant adolescents. We are actively seeking a strategy to allow inclusion of adolescents under age 18, but we do not know how long this limitation will continue.

\section{Recruitment, consent, and baseline data collection}

Regardless of study condition, each woman is assessed for eligibility after her first individual intake visit at which her health and gestational age are assessed, and laboratory and HIV tests are completed. Some of these data are used to determine eligibility (e.g., age, gestational age). Women then go through the informed consent process, sign the consent form, and then the baseline self-report survey. Refusals and reasons given by eligible women will be documented as indicators of selfselection bias.

\section{Randomization}

Using a computer program, our statistician generated randomization card sets. Each set contains randomized assignment for a cluster of 16 (8 to each study condition) or 24 women (12 to each study condition). A new packet is used with each new cluster. After completion of the baseline survey, the woman takes the next card and, until it is revealed, neither the woman nor researcher is aware of her assignment.

\section{Retention}

To maximize retention in the effectiveness evaluation across the 4 data collection time points, we use the same strategies that were successful in our pilot. When possible, we obtain cell phone numbers at which the woman can be reached as well as the location of her home. If a woman misses a data collection appointment and cannot be reached, a health surveillance assistant will go into the community and attempt to locate the woman.

\section{Sample size and power}

Sample size determination was based on power analyses for the two primary outcomes: rates of preterm birth and rates of HIV testing for women and partners (H1 \& $\mathrm{H} 2$ ). At the writing of the proposal, the preterm birth rate for Malawi was estimated to be $18 \%$ [57]. We expect that the preterm birth rate for women in the individual antenatal care will mirror the national rate. Because a US-based RCT found a 30\% reduction in the preterm birth rate for women in group antenatal care [26], we expect the preterm birth rate for women in group antenatal care in Malawi to reflect a 30\% reduction. Our pilot data showed an attrition rate of 19\% through 6 weeks postpartum; therefore, we assume a $30 \%$ attrition rate for a longer duration of 6 months follow-up. Recruitment and randomization are done in clusters and we assume a within-cluster correlation of 0.1 ; therefore, a sample size of 1776 will ensure $80 \%$ statistical power (two-sided alpha $=0.05$ ) for detecting group preterm birth rate difference after attrition (final $\mathrm{N}$ at 6 months $=$ 1244). For H2, our pilot results from Malawi showed that $51 \%$ of group antenatal care partners were tested during pregnancy versus $27 \%$ for usual care. The proposed sample size of 1776 will ensure a statistical power of $>99 \%$ for detecting similar difference. Power analyses for Aim 1 were performed via simulations in SAS for dichotomous outcomes in a multi-level design. The proposed sample of 1776 women at baseline (1244 after attrition) ensures $>80 \%$ statistical power for testing our secondary outcomes.

For our exploratory hypothesis (H4), participating clinic data for the first 6 months of 2017 showed an average HIV infection rate of $10.7 \%$. With a sample of 1776 (1244 after attrition), we estimate there will be a subsample of 133 HIV-infected women at 6 months postpartum. Only $78.8 \%$ of women diagnosed at their first antenatal care visit are retained in care 12 months later; $60 \%$ of those who get their infant tested do not return for the infant's results [59]. Our sample will not provide adequate power to determine differences in retention by model of care; however, clinically important information about group antenatal care's effect on continuation of care for HIV-infected women and infant follow-up through 6 months postpartum will be beneficial.

\section{Aim 1 Effectiveness study conditions}

The care for women in the RCT differs from national antenatal care because usual antenatal care (control condition) consists of 8 antenatal care contacts aligning with the 2016 WHO recommendations [24]. The postnatal care schedule is unchanged with the expectation of visits with $24 \mathrm{~h}, 1$ week, and 6 weeks postpartum. Women, regardless of study condition, are offered the same intake visit and 8-visit antenatal care schedule. The two study conditions are described below:

- Control condition, Individual care: Women listen to a health lecture and are provided antenatal and postnatal care services on a first come, first served basis. They meet individually with a midwife for a physical assessment. Women complete laboratory tests (including HIV testing) at their intake (first) visit. 
- Intervention condition, Group care: Women have the same number of visits as those in usual care at the same time points in their pregnancy and after delivery. The intake visit ends with assignment to group care and each woman is given her group appointment schedule. All subsequent antenatal care visits occur as a 2 -h group visit with the same women and co-facilitators, one midwife and one community volunteer. The 1 -week postpartum visit is an individual one because of the short interval between delivery and the recommended visit. Women in the group will deliver over the course of about 4 weeks, so it is not feasible to have a group visit at the 1 -week time point. The second postnatal visit at 4-8 weeks after delivery is a 2 -h group visit.

\section{Intervention: 3-step sequence for implementation}

Following a model that was used to successfully scale-up Kangaroo Mother Care in South Africa, each clinic uses a 3-step sequence for implementation: prepare, rollout, and sustain (6 months with support and 6 months independently). We purposely staggered rollout to ensure that our implementation team is able to provide intensive support and interactive assistance as each clinic begins to offer group antenatal care. The three steps are described in more detail in the Procedures section and summarized in Fig. 1.

\section{Prepare (2 months)}

Together with our implementation team, each clinic develops and presents a plan to fit their clinic's context and sends future group care facilitators (providers and community volunteers) to a training workshop.

\section{Rollout}

Each clinic enrolls equal numbers of group and individual antenatal care participants in clusters for the effectiveness study (Aim 1).

\section{Sustain (12 months)}

With support and assistance from the implantation team, the clinic will review their experiences and the available evidence to decide whether to continue offering group antenatal care at their clinic.

Intervention: group antenatal care implementation toolkit Our evidence-based group antenatal care Toolkit provides clinics with all of the information and materials needed to implement group antenatal care [60-62]. The Toolkit includes a clinic implementation guide, group antenatal care training and facilitation guide, benchmarks, a training video, and interactive learning materials. Although the Toolkit can be used alone, research shows that implementation proceeds more quickly and with greater fidelity when supportive consultation and interactive assistance is provided [62-66]. To help with planning and resolving emerging challenges, our implementation team provides intensive and supportive consultation and interactive assistance each week as each clinic prepares and during the first 2 months of rollout of the group antenatal care model. We work with clinics as they adapt group antenatal care to their context (e.g. considerations may include staffing levels, busiest days, client preferences). We ensure that adaptions will not threaten fidelity. Thereafter, we meet monthly and then during sustain, quarterly. Clinics are welcome to call between meetings to discuss emergent problems. Experiences, challenges, and solutions will later be compiled and shared in a "Lessons Learned" chapter that will be added to the implementation guide.

Given there were no experienced group antenatal care trainers in Malawi, two experienced CenteringPregnancy trainers from the US led the 4-day training workshop. The workshops are appropriate for both midwives and the community volunteers with lower literacy and designed to provide opportunities for experiential learning and skills-building. After co-facilitators at clinics 1-3 have gained substantial experience, we will identify those who would like to become group antenatal care master trainers. The US trainers will then offer an Advanced Training Workshop in Year 3 to develop these skills. Master trainers will then gain experience because they will lead the Year 3 training workshop for clinics 3-6. This builds capacity since a local cadre of group

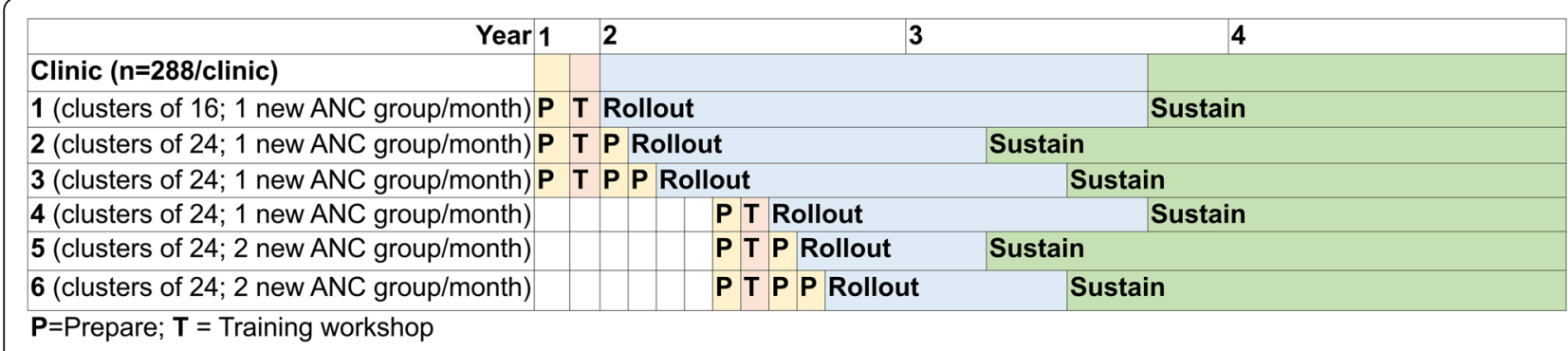

Fig. 1 Timeline showing the 3-Step Implementation Model used by each clinic 
antenatal care master trainers will be available to provide future trainings and implementation support.

\section{Measures}

Aim 1 outcomes evaluating the impact of group antenatal care are measured at four time points (T1: baseline, T2: late pregnancy, and T3 and T4, 2- and 6months postpartum). Data are produced from self-report surveys, health records extractions, and two biomarkers assessing anemia (T1-T4) and pregnancy status (T4). Survey measures were selected from those used in our pilot and most have been used with similar clinical populations and showed high reliability (Table 2; Additional File 1). Pregnancy knowledge and behaviors indices have been modified to reflect expansion from 4 to 8 visits and behavioral recommendations. These items were reviewed by a panel of expert midwives for content validity. Variables examined at 6 months after delivery are assessed using standard measures.

\section{Aim 2 Study population (implementation)}

Data for the implementation portion of the study are drawn from key stakeholders at the Ministry of Health, District Health Office, clinic administration, and cofacilitators (antenatal care midwives (and master trainers) and community volunteers). Eligibility criteria are based on the person's position at the time of data collection (e.g., Director of the Reproductive Health Unit, midwife at participating clinic). Consent is obtained before data are collected for the first time.

Table 2 Group antenatal care effectiveness outcomes (Aim 1)

\begin{tabular}{|c|c|c|c|c|c|c|}
\hline \multirow[t]{2}{*}{ Construct } & \multirow[t]{2}{*}{ Operational Measure } & \multicolumn{4}{|c|}{ Time } & \multirow[t]{2}{*}{ Source $^{a}$} \\
\hline & & 1 & 2 & 3 & 4 & \\
\hline \multicolumn{7}{|c|}{$\mathrm{H} 1$. Less prematurity and mortality } \\
\hline Preterm Birth (Prim) & $<37$ weeks gestational age & & & $\cdot$ & & $\mathrm{H}, \mathrm{S}$ \\
\hline Spontaneous abortion & Pregnancy loss $<20$ weeks & & & $\cdot$ & & $\mathrm{H}, \mathrm{S}$ \\
\hline Stillbirth & Baby born with no signs of life $\leq 28$ weeks gestation [67] & & & $\cdot$ & & $\mathrm{H}, \mathrm{S}$ \\
\hline Low birthweight & $<2500 \mathrm{~g}$, measured within $24 \mathrm{~h}$ of birth & & & $\cdot$ & & $\mathrm{H}, \mathrm{S}$ \\
\hline Neonatal death & Newborn dies with 28 days after birth & & & $\cdot$ & $\cdot$ & $\mathrm{H}, \mathrm{S}$ \\
\hline Maternal mortality & Death in pregnancy or $\leq 42$ days of end of pregnancy & & $\cdot$ & $\cdot$ & & $\mathrm{H}, \mathrm{S}$ \\
\hline \multicolumn{7}{|c|}{ H2. More positive HIV prevention outcomes } \\
\hline Partner HIV Test (Prim) & Proportion of partners tested during pregnancy & & $\cdot$ & & & S \\
\hline Women, HIV Test & Initial HIV test; If seronegative, repeated in 3rd trimester & $\cdot$ & $\cdot$ & & & \\
\hline HIV Knowledge & General knowledge (5 items) & $\cdot$ & $\cdot$ & & & \\
\hline PMTCT Knowledge & Mother-to-child transmission (4 items) & $\cdot$ & $\cdot$ & & & \\
\hline Sexual Health Behaviors & Condom use; Partner communication (5 yes/no items) & $\cdot$ & $\cdot$ & $\cdot$ & $\cdot$ & $\mathrm{S}$ \\
\hline \multicolumn{7}{|c|}{ H3. Optimal secondary outcomes } \\
\hline \multirow[t]{2}{*}{ Antenatal \& postnatal care } & Attendance; birth in a health facility (yes/no) & & & $\cdot$ & & $\mathrm{H}, \mathrm{S}$ \\
\hline & 21 services received; 18 educational topics (modified) & & $\cdot$ & & & \\
\hline Satisfaction with Care & 10-items; 5-point Likert scale; Range 10-50, $a=0.980$ & & $\cdot$ & & & $S$ \\
\hline Pregnancy Knowledge & 25 items based on content (modified for this study) & $\cdot$ & $\cdot$ & & & $\mathrm{S}$ \\
\hline Healthy Behaviors & 14 recommended behaviors (modified for this study) & $\cdot$ & $\cdot$ & & & $\mathrm{S}$ \\
\hline Mental Distress & Self Reporting Questionnaire [68-71]; 20 items; $a=0.789$ & $\cdot$ & $\cdot$ & $\cdot$ & $\cdot$ & $\mathrm{S}$ \\
\hline Anemia & Hemoglobin (Hb), HemoCue ${ }^{\oplus}(\mathrm{Hb}<11 / / \mathrm{dL})[72]$ & $\cdot$ & $\cdot$ & $\cdot$ & - & $\mathrm{T}$ \\
\hline Hypertension & Blood pressure changes and symptoms & $\cdot$ & $\cdot$ & $\cdot$ & & $\mathrm{H}, \mathrm{S}$ \\
\hline Postpartum bleeding & Bleeding (> $500 \mathrm{ml})$; hemorrhage (> $1000 \mathrm{ml}$ ) & & & $\cdot$ & & $\mathrm{H}, \mathrm{S}$ \\
\hline Exclusive Breastfeeding & Exclusive breastfeeding duration, \# days & & & & $\cdot$ & $\mathrm{S}$ \\
\hline Family Planning & Using a family planning method & & & & $\cdot$ & $\mathrm{S}$ \\
\hline Early repeat pregnancy & Negative test and no reported pregnancy loss & & & & $\cdot$ & $\mathrm{S}, \mathrm{T}$ \\
\hline \multicolumn{7}{|c|}{ H4. Exploratory: Successful transition in continuum of HIV care (subset of HIV-infected women) } \\
\hline Women & Received ART medication & $\cdot$ & $\cdot$ & $\cdot$ & $\cdot$ & $\mathrm{S}$ \\
\hline Infant & Infant HIV test, status known & & & $\cdot$ & - & $\mathrm{H}, \mathrm{S}$ \\
\hline
\end{tabular}

${ }^{a}$ Source: $H$ Health record, $S$ Self-report survey, $T$ Medical test 
Persons no longer in the designated position are dropped, and persons coming into these positions are consented to participate at their first data collection.

Our Aim 2 degree of implementation success measures are in Table 3. Three indicators (continuation, reach and fidelity) are assessed after 12 months of sustaining. For clinics that continue to offer group care, degree of success is examined using a combination of reach and fidelity indicators and the relationship between reach and fidelity will be explored in our analysis. We use the same fidelity observation procedure and data collection instrument used in our pilot (Additional File 2). Guided by the 5 domains of the consolidated framework for implementation research (CFIR), contextual factors are events, situations, and clinic contextual factors that are abstracted from study notes, interview data, and staff surveys (Additional file 3) [73, 79, 80].

\section{Procedures}

As described above, each clinic follows the same sequence of steps to Prepare, Rollout and Sustain (6 months with support, 6 months independently) group antenatal care. We staggered the initiation of group antenatal care to allow the implementation team (not blinded to study condition) provide intensive Interactive Assistance. During rollout, the effectiveness team (blinded to study condition) leads the collection of effectiveness data at the four time points during pregnancy (T1 and T2) and postpartum (T3 and T4).

\section{Aim 1 Effectiveness procedures}

Clusters of 24 women are currently being enrolled with 12 randomly assigned to each study condition (Fig. 1). The clinic with the lowest patient volume enrolls cohorts of 16 women with 8 assigned to each study condition which extends their time to complete rollout. Before randomization and to promote privacy and better reporting of sensitive information each woman completes a baseline self-report survey administered off-line using ACASI [81].

To manage timely data collection, a spreadsheet lists all participants as they enroll, along with ID numbers, clinic, contact information, enrollment date, and weeks gestation at entry. After each day's data collection, the team enters the date information was obtained or, if planned data collection was not completed, the reason (e.g., no show, in-hospital etc.) and planned follow-up action and date. Each week the list of women needing follow-up, type of follow-up and their contact information is generated to guide the data collection schedule. At weekly effectiveness team meetings, recruitment plans and the spreadsheet identifying follow-up data collection needs is presented. The team reviews this and makes assignments. The team discusses challenges; ideas for reaching women lost to follow-up are discussed; and plans for the next week are made.

A team member calls each woman to schedule the data collection appointment and offer reminders. If a woman does not have a cell phone, health surveillance assistants from the clinic will go to the woman's home to make the appointment. Women's hemoglobin $(\mathrm{Hb})$ levels are tested at all 4 time points using a simple point-of-care blood droplet test, following standard procedures provided by the manufacturer to ensure validity [72]. At the 4th survey, each woman is asked to take the pregnancy urine test kit in a clinic restroom. A team member will review the results with her and record the result. We discard all biomarkers materials in our trash container, which is removed at the end of the day and disposed of in the clinic incinerator. For women who were identified as HIV+ at intake or during pregnancy, maternal and infant HIV clinic attendance and infant HIV status at 6 months will be verified from medical records. Shortly after the expected delivery date or early loss, our data clerks will work with clinic staff to search delivery records to verify fetal loss, stillbirth, or live birth plus prematurity and birthweight. Most women deliver at the clinic where they obtain care, but if a woman has complications in pregnancy or delivery, she is sent to a referral hospital. After a woman gives birth, a research team member will obtain these data to capture the birth outcomes from records. $\mathrm{Hb}$ values, pregnancy test results, and health records will be recorded by the research team in the participant's Health Passport (as appropriate) as well as on data collection forms developed for the research project, using the participant's project ID code.

\section{Aim 2 Implementation procedures}

As described above, over the course of 3 years, each of the six clinics will prepare, rollout, and sustain group care. Rollout is staggered to ensure that the implementation team can provide intensive support and interactive assistance. The procedures of the 3 steps of the implementation model are described in detail below.

\section{Prepare (2 months)}

Our implementation team sensitizes each clinic to group antenatal care. Each clinic conducts a group antenatal care walk-through, identifies who will coordinate group antenatal care, identifies community volunteers, adapts the plan to fit their clinic's context, presents their plan at a clinic meeting, and sends providers and community volunteers who will be co-facilitators to a group antenatal care training workshop.

Rollout is the period in which the Aim 1 Effectiveness component is conducted. Each clinic enrolls equal numbers of group and individual antenatal care participants in clusters for the effectiveness study. The evaluation 
Table 3 Measures and data sources for degree and variation of implementation success (Aim 2)

\begin{tabular}{|c|c|c|}
\hline \multicolumn{2}{|c|}{ Indicators based on data from the last quarter of sustain independently } & \multirow{2}{*}{$\frac{\text { Source }}{\text { Benchmarks }}$} \\
\hline Continuation & Yes/No indicating ceased or continued to offer group antenatal care & \\
\hline Reach & $\%$ in group care based on the total number of antenatal care clients & Benchmarks \\
\hline Fidelity & $\begin{array}{l}\text { Quarterly clinic mean scores; High fidelity indicated by high group engagement, high session management and } \\
\text { interpersonal facilitation skills (e.g., controlling negativity, drawing out participants), and overall rating as more like } \\
\text { discussion than classroom. }\end{array}$ & Observations \\
\hline \multicolumn{3}{|c|}{ Contextual Factors by CFIR Domain [73] (ongoing) } \\
\hline \multirow[t]{3}{*}{ Intervention } & - Time: Group vs. usual time required to deliver antenatal care & \multirow{3}{*}{$\begin{array}{l}\text { Interviews } \\
\text { Study Notes }\end{array}$} \\
\hline & - Additional group care expenses: training, equipment and supplies & \\
\hline & - Other: factors affecting implementation (space, scheduling) & \\
\hline $\begin{array}{l}\text { Outer } \\
\text { Setting }\end{array}$ & $\begin{array}{l}\text { - Ministry of Health (annually); District Health Office (annually); Safe Motherhood Task Force (Quarterly); Other } \\
\text { stakeholders, as needed }\end{array}$ & $\begin{array}{l}\text { Meetings } \\
\text { Interviews }\end{array}$ \\
\hline Inner Setting & $\begin{array}{l}\text { - Clinic characteristics and events reported (staff transfers, leadership, interactions) } \\
\text { - Successes, challenges, solutions, decisions, interpersonal interactions, and evaluation of evidence; coded as positive, } \\
\text { negative or mixed }\end{array}$ & $\begin{array}{l}\text { Benchmarks } \\
\text { Interviews } \\
\text { Observation }\end{array}$ \\
\hline \multirow{4}{*}{$\begin{array}{l}\text { Individual } \\
\text { (Staff) }\end{array}$} & - Basic demographics, including education level and experience & \multirow[t]{4}{*}{ Survey } \\
\hline & - Maslach Burnout Inventory (9 items; $a>$.79) [74-76]; & \\
\hline & • Health Worker Motivation (10-item; range 10-50) [77, 78]; & \\
\hline & - Perspectives on group care (5 items) at baseline and beginning and end of sustain & \\
\hline \multirow[t]{2}{*}{ Process } & $\begin{array}{l}\text { - Quarterly Benchmark Scores: } 25 \text { yes/no items (1 point for each yes). Integrates co-facilitators' self-evaluation of fidelity } \\
\text { and time records. Patterns over time: Fail; Interrupted; Sporadic; Continuous }\end{array}$ & $\begin{array}{l}\text { Benchmarks } \\
\text { Self- } \\
\text { evaluations }\end{array}$ \\
\hline & - Fidelity: Quarterly ratings & Observations \\
\hline
\end{tabular}

team completes consent, baseline data collection, and random assignment and then provides the clinic with the lists of women randomly assigned to group antenatal care. During rollout, providers can call our team for advice as needed to address any unforeseen barriers/issues.

Sustain is a 12-month process that begins with a clinic decision to continue offering group antenatal care during the month that the last cluster of women is enrolled in the effectiveness study. The co-facilitators' experience and feedback is presented to clinic administration and staff so that decisions can be made about whether to continue offering group antenatal care. If so, they also determine if they need to train additional facilitators and the number of groups they will enroll each month. To build capacity, we treat the first 6 months of sustain as transitional and continue to offer our support and assistance. The clinic continues to receive interactive assistance from the implementation team and completes benchmarks each quarter. If they decide not to enroll new groups, they complete care for women already enrolled and cease participation.

After another 6 months, the clinic will again review their experiences and the available evidence to decide whether to continue offering group antenatal care at their clinic. Clinics will continue to complete and share their benchmarks, and an implementation team member will make a brief quarterly check-in to pick up a copy of the benchmarks and consult. If training is needed, the clinic will contact a Malawian master trainer to arrange. Some clinics may elect to not continue offering group antenatal care and others may flounder and find themselves unable to continue. Thus, some clinics may not complete 12 months of sustaining. We will assess their 'end point' as when they no longer form groups.

\section{Data analysis \\ Data management}

Project protocols promote proper and timely preparation of data for analysis and secure data storage. All data are identified by location, date and clinic. A unique individual code is assigned to individual-level data. Signed consent forms and a master list linking names and code numbers are securely stored in locked cabinets separately from data. Data are transferred to UIC using secure cloud storage and strict confidentiality guidelines for HIPAA compliance and data confidentiality guidelines for Malawi.

\section{Periodic clinic reports}

The 6 clinics roll out at different time points and require their own clinic data so they can assess the impact of group antenatal care for their own clients. Every 6 months, our team will generate a descriptive report summarizing outcomes for group and individual care by each clinic and totals for all clinics, using the cumulative 
data available. Reports will also be shared with the Ministry of Health's Safe Motherhood Task Force.

\section{Aim 1 Statistical analyses}

The goal is to evaluate the overall effectiveness of group antenatal care compared to individual care. In preliminary analyses, we will complete a CONSORT diagram of recruitment, retention, and loss to follow-up from initial recruitment through final data collection. We will determine retention rates and examine attrition bias, e.g., significant demographic differences between those retained and those lost to follow-up using Cox proportional hazards regression for time-to-dropout. Randomization success will be evaluated by comparing group differences in baseline demographics and measures of interest such as HIV testing, pregnancy knowledge and behaviors, etc. Any significant differences in sites' demographic characteristics at baseline and attrition biases will be controlled in later analyses. Bivariate analyses between the outcome variables and study group will first be conducted using t-tests (continuous variables) or Chi-squared tests. Potential clinical site differences will be identified using Analysis of Variance (ANOVA) models for continuous variables or Chi-squared tests for categorical variables.

All multivariate analyses will be conducted using multi-level hierarchical models, with clinics entered in models as both fixed effects (to adjust for mean site differences) and random effects (to account for within-site correlations). For outcomes that are measured only once, mixed-effects linear regression (continuous), Poisson regression (count data), or logistic regression (dichotomous or ordinal) models will employed. Intra-class (site) correlation coefficient will be estimated. Group assignment, sites, women's demographics, and obstetric characteristics will be included as fixed effects. Potential effect modifiers will be identified (based on theoretical reasons) by testing the interaction between group and the potential modifier. For continuous outcomes that are measured more than once, such as mental distress or partner communication, mixed-effect regression models will be employed to examine group effect over time with both random site and individual (nested within site) effects. Generalized Estimating Equation models will be employed to analyze dichotomous repeated outcomes (such as consistent condom use in the last 2 months). In all repeated measures models, the interaction between group and time will be the parameter of interest, and time-point specific group difference will be estimated from the model. In all multivariate models, backward selection methods will be employed to select significant factors that are associated with the outcome. All statistical tests will be two-sided tests, controlling for probability of Type I error of 0.05 .

\section{Aim 2 Mixed-methods analyses}

Addressing Aim 2 is an iterative process that begins early and continues throughout the study. We extract contextual factors (events or statements) from study notes, interviews, and observations. We focus on reported successes, challenges, solutions tried and whether successful, as well as champions and naysayers. We will then categorize events or statements according to CFIR domains and constructs.

Data will be coded separately by 2 coders who will consult with the team to review discrepancies, refine code definitions, and recode until intercoder reliability exceeds $85 \%$. In addition to the CIFR, new themes may emerge; these will be developed into new codes and discussed in team meetings. Final codes will be compiled in the master codebook and applied to the coding of qualitative data [79], [82].

From the last quarter of sustain for each clinic, we will determine the degree of success using 3 indicators (Table 3). We will use data from the last quarter of sustain, because this time interval reflects each clinic's performance when sustaining independently. The minimum indicator of implementation success will be whether a clinic continues to offer group antenatal care or not. For clinics that continue, we will base degree of success on a combination of reach and fidelity and compare clinics using analysis of variance. Together these indicators will allow us to categorize the 6 clinics according to their degree of success. We cannot know what this will look like beforehand, but we anticipate 2 or 3 major categories (e.g. successful or not, or high, medium, and low).

As each clinic completes the sustain step, we will begin to describe implementation processes and factors related to prepare, rollout and sustain and their associations with degree of implementation success. Using a team-based case study approach, we will rate each construct according to whether it facilitated, hindered, or did not affect implementation [79]. The end product will be a case summary for each clinic [83, 84]. Our quantitative measures (i.e., staff burnout and motivation and fidelity scores) will first be analyzed using standard statistical procedures. For mixed methods analyses, these quantitative measures will be transformed into interval or dichotomous categories.

Using well-established mixed methods analyses, [85, 86] we will then compare across cases to identify constructs most strongly related to degree of implementation success (variable-oriented approach) [84]. Our mixed method analyses to construct the final across case matrix will also be guided by the procedures used by Damschroder and Lowery to identify contextual factors associated with greater or less implementation success for an obesity program in the US [83]. At the conclusion of Aim 2 analyses, we will identify the contextual factors 
that consistently relate to degree of implementation success (or failure) across all 6 clinics.

\section{Study status}

At the time this manuscript was submitted for publication, the study was underway. Three clinics have completed the prepare step. The first group antenatal care training workshop was successful; 38 midwives and 9 community volunteers were trained. A total of 17 clusters of women are enrolled from three clinics $(n=352)$.

\section{Discussion}

This is the first individually randomized RCT of group antenatal care in a low-income African country that has high fidelity to CenteringPregnancy, the only evidencebased group antenatal care model, and incorporates the 2016 WHO recommendations for eight antenatal contacts with woman-centered care. At the conclusion of this five-year study, we will be able to assess whether an eight-contact model meets women's needs and if group antenatal care is equal to or better than the usual individual care model.

In addition to meeting WHO's call for the rigorous study of group antenatal care, our effectivenessimplementation hybrid type 1 design also allows us to make an important contribution to implementation science. We incorporate process evaluation within the randomized trial so that we can systematically identify factors that impede and facilitate successful implementation over time. Implementation of an evidence-based model with fidelity (i.e., adherence to and high-quality delivery of the core components) can be difficult in the best of circumstances. Midwives in Malawi and other poorly-resourced health systems with a shortage of providers may lack the capacity for evidence-based implementation [87]. We are facilitating replication through careful attention to fidelity, which is supported by the training workshop, an implementation toolkit, and technical assistance. We will document if implementation of the group antenatal care model at each clinic and over time is faithful to core components of the intervention. These data will be of interest to those implementing other evidence-based interventions in low-resource healthcare settings [88].

Although the well-established CIFR has been used once retrospectively in a low-resource setting, to the best of our knowledge, this is the first study to use CFIR prospectively to describe implementation factors in a lowresource country [73]. Findings from this evaluation will contribute to the growing body of evidence identifying key factors positively and negatively affecting implementation, with special relevance for low-resource settings. If effectiveness is demonstrated, the implementation evaluation will be critical to and facilitate national scale-up this paradigm-changing and transformative model that aims to improve antenatal care quality.

Group antenatal care has been shown to improve the quality of care and maternal and infant outcomes in the US. The implementation of group antenatal care in a context of high mortality and HIV prevalence is especially innovative because this is a comprehensive intervention that simultaneously addresses the multiple needs of women. In addition to saving lives by reducing rates of preterm birth and low birth weight, data show that investing in quality antenatal care provides a return on investment through health expenditure savings. In Malawi and many other lower income countries, nearly every woman will attend antenatal care several times over her life. Half of Malawian women have begun childbearing by age 19, and women have an average of 4.4 births [89]. If, in this context, group antenatal care can reduce risk for preterm birth, impact HIV prevention, including reducing new infections and prevention of mother-to-child transmission, improve infant feeding practices, and increase birth spacing, the population health impact will be enormous. If effective, it is also possible that higher quality of care may increase attendance and/or overload a clinic if the demand for services increases. Policy makers will have to consider the longterm population health cost savings that accrue from having healthier mothers, infant, and families and find ways to creatively support the education of more midwives to optimize antenatal care. This study will provide rigorous evidence documenting whether group antenatal care is effective and should be scaled up. If our study shows positive health impacts in Malawi, the results will provide strong evidence and valuable lessons learned for widespread scale-up other low-resource settings.

\section{Supplementary information}

Supplementary information accompanies this paper at https://doi.org/10. 1186/s12889-020-8276-x

Additional file 1: Survey items and associated time points

Additional file 2: Fidelity and Self-Evaluation Forms

Additional file 3: Benchmark Scoring Sheet

\section{Abbreviations}

ACASI: Audio Computer-Assisted Self-Interview software; CFIR: Consolidated Framework for Implementation Research; RCT: Randomized Controlled Trial; WHO: World Health Organization

\section{Acknowledgements}

We wish to express our appreciation to the Malawi Ministry of Health for continued efforts to improve the public healthcare system in Malawi. A special thanks to Sharon Rising and her colleagues at Group Care Global and Amy MacDonald for partnering with our team. We are inspired by the midwives, community volunteers, and administrators at participating clinics because they show that they are not only committed to serving their patients, but also and dedicated to improving reproductive, maternal, newborn, and child health outcomes through implementation of evidencebased practice. We are indebted to the women who agreed to participate in 
this study and appreciate the time and effort involved in moving science forward.

\section{Authors' contributions}

CLP, KFN, LLM, and EC conceived the study. LL conducted the power analysis and condensed it for this manuscript. All authors contributed to the study protocol and its refinement. AG and ETA wrote the first manuscript draft incorporating team modifications. All authors edited and revised manuscript draft. All authors reviewed and approved the final manuscript draft.

\section{Funding}

The research study is funded by the National Institutes of Health, National Institute for Nursing Research, number. The funders played no role in research design, data collection, data analysis, manuscript write-up, or decision to publish. Any opinions, findings, conclusions, or recommendations expressed in this article are those of the authors alone and do not necessarily reflect the views of the National Institutes of Health.

\section{Availability of data and materials}

De-identified survey data will be made available by emailing a request to: cpatil@uic.edu.

We will not provide full transcriptions of qualitative data as these may contain information that could compromise identity.

\section{Ethics approval and consent to participate}

The Institutional Review Board (IRB) at the University of Illinois at Chicago (\#2018-0845) and University of Malawi College of Medicine Research Ethics Committee (\#P.10/18/2498) approved this study. We obtain written informed consent from all study participants.

\section{Consent for publication}

Not applicable.

\section{Competing interests}

KFN serves as a volunteer member of the Advisory Board for Group Care Global, a non-profit organization dedicated to promoting a group care model based on the CenteringPregnancy; there is no compensation for this service. The other authors declare that they have no competing interests.

\section{Author details}

${ }^{1}$ University of Malawi, Kamuzu College of Nursing, PO Box 415, Blantyre, Malawi. ${ }^{2}$ Johns Hopkins University, School of Nursing, 525 North Wolfe Street, Baltimore, MD 21205, USA. ${ }^{3}$ University of Illinois at Chicago, School of Public Health, 1603 W. Taylor Street (M/C 932), Chicago, IL 60612, USA. ${ }^{4}$ University of Illinois at Chicago, College of Nursing, 845 S. Damen Avenue (M/C 806), Chicago, IL 60612, USA.

Received: 15 January 2020 Accepted: 27 January 2020

Published online: 10 February 2020

\section{References}

1. WHO, UNICEF, UNFPA Group, World Bank Division, UNDP. WHO | Maternal Mortality: Levels and Trends. World Health Organization; 2019.

2. Say L, Chou D, Gemmill A, et al. Global causes of maternal death: A WHO systematic analysis. Lancet Glob Health. 2014;2(6). https://doi.org/10.1016/ S2214-109X(14)70227-X.

3. Kassebaum NJ, Barber RM, Dandona L, et al. Global, regional, and national levels of maternal mortality, 1990-2015: a systematic analysis for the global burden of disease study 2015. Lancet. 2016;388(10053):1775-812. https://doi. org/10.1016/S0140-6736(16)31470-2

4. UNICEF. Levels \& Trends in Child Mortality Report 2019.; 2019.

5. Lawn JE, Blencowe $\mathrm{H}, \mathrm{Oza} \mathrm{S}$, et al. Every newborn: progress, priorities, and potential beyond survival. Lancet. 2014;384(9938):189-205. https://doi.org/ 10.1016/S0140-6736(14)60496-7.

6. Lawn JE, Kinney MV, Belizan JM, et al. Born too soon: accelerating actions for prevention and care of 15 million newborns born too soon. Reprod Health. 2013. https://doi.org/10.1186/1742-4755-10-S1-S6.

7. Kinney MV, Kerber KJ, Black RE, et al. Sub-Saharan Africa's mothers, newborns, and children: where and why do they die? PLoS Med. 2010;7(6): e1000294. https://doi.org/10.1371/journal.pmed.1000294.
8. Friberg IK, Kinney MV, Lawn JE, et al. Sub-Saharan Africa's mothers, newborns, and children: how many lives could be saved with targeted health interventions? PLoS Med. 2010;7(6):e1000295. https://doi.org/10.1371/ journal.pmed.1000295.

9. Requejo $\mathrm{JH}$, Newby $\mathrm{H}$, Bryce J, et al. Measuring Coverage in $\mathrm{MNCH}$ : Challenges and Opportunities in the Selection of Coverage Indicators for Global Monitoring. Madise N, ed. PLoS Med. 2013;10(5):e1001416. https://doi. org/10.1371/journal.pmed.1001416.

10. von Both C, Flessa S, Makuwani A, Mpembeni R, Jahn A. How much time do health services spend on antenatal care? Implications for the introduction of the focused antenatal care model in Tanzania. BMC Pregnancy Childbirth. 2006;6:22 http://ukpmc.ac.uk/abstract/MED/16796749.

11. Conrad P, De Allegri M, Moses A, et al. Antenatal Care Services in Rural Uganda: missed opportunities for good-quality care. Qual Health Res. 2012. https://doi.org/10.1177/1049732311431897.

12. Koblinsky M, Moyer CA, Calvert C, et al. Quality maternity care for every woman, everywhere: a call to action. Lancet. 2016;388(10057):2307-20. https://doi.org/10.1371/journal.pone.0160562.

13. UNAIDS. Miles to Go: Closing Gaps, Breaking Barriers, Righting Injustices |UNAIDS.; 2018. https://www.unaids.org/en/resources/documents/2018/ global-aids-update. Accessed 20 Nov 2019.

14. Nachega JB, Uthman OA, Anderson J, et al. Adherence to antiretroviral therapy during and after pregnancy in low-income, middle-income, and high-income countries: a systematic review and meta-analysis. AIDS. 2012; 26(16):2039-52. https://doi.org/10.1097/QAD.0b013e328359590f.

15. Steegers EA, von Dadelszen P, Duvekot JJ, Pijnenborg R. Pre-eclampsia. Lancet. 2010;376(9741):631-44. https://doi.org/10.1016/S01406736(10)60279-6.

16. Hodgins S, Tielsch J, Rankin K, et al. A New Look at Care in Pregnancy: Simple, Effective Interventions for Neglected Populations. Gebhardt G, ed. PLoS One. 2016;11(8):e0160562. https://doi.org/10.1371/journal.pone. 0160562.

17. Haas AD, Msukwa MT, Egger M, et al. Adherence to Antiretroviral Therapy During and After Pregnancy: Cohort Study on Women Receiving Care in Malawi's Option B+ Program. Clin Infect Dis. 2016;63(9):ciw500. https://doi. org/10.1093/cid/ciw500.

18. Kruk ME, Kujawski S, Moyer CA, et al. Next generation maternal health: external shocks and health-system innovations. Lancet. 2016;388(10057): 2296-306. https://doi.org/10.1016/S0140-6736(16)31395-2.

19. Kruk ME, Mbaruku G, McCord CW, Moran M, Rockers PC, Galea S. Bypassing primary care facilities for childbirth: a population-based study in rural Tanzania. Health Policy Plan. 2009;24(4):279-88. https://doi.org/10.1093/ heapol/czp011.

20. Olsen $\varnothing E$, Ndeki SS, Norheim OF, et al. Human resources for emergency obstetric care in northern Tanzania: distribution of quantity or quality? Hum Resour Health. 2005;3(1):5. https://doi.org/10.1186/1478-4491-3-5.

21. Leonard KL, Mliga GR, Haile MD. Bypassing health Centres in Tanzania: revealed preferences for quality. J Afr Econ. 2002;11(4):441-71. https://doi. org/10.1093/jae/11.4.441.

22. Downe S, Finlayson K, Tunalp O, Metin GA. What matters to women: a systematic scoping review to identify the processes and outcomes of antenatal care provision that are important to healthy pregnant women. BJOG An Int J Obstet Gynaecol. 2016;123(4):529-39. https://doi.org/10.1111/ 1471-0528.13819.

23. Badal NF, Yusuf UA, Egal J, et al. With knowledge and support, women can attend antenatal care: The views of women in IDP camps in Somaliland. Afr J Midwifery Womens Health. 2018;12(3):138-43. https://doi.org/10.12968/ ajmw.2018.12.3.138.

24. World Health Organization. WHO recommendations on antenatal Care for a Positive Pregnancy Experience. Geneva: World Health Organization; 2017. http://apps.who.int/iris/bitstream/10665/250796/1/9789241549912-eng. pdf?ua=1. Accessed 21 Nov 2016

25. Rising SS, Quimby $\mathrm{CH}$. The CenteringPregnancy model: the power of group health care. New York: Springer Publishing Company; 2016.

26. Ickovics JR, Kershaw TS, Westdahl C, et al. Group prenatal care and perinatal outcomes a randomized controlled trial. Obstet Gynecol Surv. 2007;110(2 Pt 1):330-9. https://doi.org/10.1097/01.AOG.0000275284.24298.23.

27. Gareau S, Lòpez-De Fede A, Loudermilk BL, et al. Group prenatal care results in Medicaid savings with better outcomes: a propensity score analysis of CenteringPregnancy participation in South Carolina. Matern Child Health J. 2016;20(7):1384-93. https://doi.org/10.1007/s10995-016-1935-y. 
28. Ickovics JR, Reed E, Magriples U, Westdahl C, Schindler Rising S, Kershaw TS. Effects of group prenatal care on psychosocial risk in pregnancy: results from a randomised controlled trial. Psychol Health. 2011;26(2):235-50. https://doi.org/10.1080/08870446.2011.531577.

29. Ickovics JR, Earnshaw V, Lewis JB, et al. Cluster randomized controlled trial of group prenatal care: perinatal outcomes among adolescents in New York City health centers. Am J Public Health. 2016;106(2):359-65. https://doi.org/ 10.2105/AJPH.2015.302960.

30. Crockett A, Heberlein EC, Glasscock L, Covington-Kolb S, Shea K, Khan IA. Investing in CenteringPregnancy ${ }^{\mathrm{TM}}$ group prenatal care reduces newborn hospitalization costs. Womens Health Issues. 2017;27(1):60-6. https://doi.org/ 10.1016/j.whi.2016.09.009.

31. Novick G, Reid AE, Lewis J, Kershaw TS, Ickovics JR, Rising SS. Group Prenatal Care: Model Fidelity and Outcomes. Am J Obstet Gynecol. 2013;209(2):112. e1-6 http://linkinghub.elsevier.com/retrieve/pii/S0002937813003001.

32. Patil C, Abrams E, Klima C, et al. CenteringPregnancy-Africa: a pilot of group antenatal care to address millennium development goals. Schindler RS, Quimby CH, eds. Midwifery. 2013;29(10):1190-8. https://doi.org/10.1016/j. midw.2013.05.008.

33. Patil CL, Klima CS, Steffen AD, Leshabari SC, Pauls H, Norr KF. Implementation challenges and outcomes of a randomized controlled pilot study of a group prenatal care model in Malawi and Tanzania. Int J Gynecol Obstet. 2017;139(3):290-6. https://doi.org/10.1002/ijgo.12324.

34. Patil CL, Klima CS, Leshabari SC, et al. Randomized controlled pilot of a group antenatal care model and the sociodemographic factors associated with pregnancy-related empowerment in sub-Saharan Africa. BMC Pregnancy Childbirth. 2017;17. https://doi.org/10.1186/s12884-017-1493-3.

35. Norr K, Patil C. Adapting CenteringPregnancy-based group antenatal care globally. In: Schindler RS, Quimby CH, editors. The CenteringPregnancy model: the power of group health care. New York: Spring Publishing Company; 2016. p. 223-51.

36. Patil C, Norr K. Field notes: Malawi and Tanzania. In: Schindler RS, Quimby $\mathrm{CH}$, editors. The CenteringPregnancy model: the power of group health care. New York: Springer Publishing Company; 2016. p. 302-8.

37. Lori JR, Munro ML, Chuey MR. Use of a facilitated discussion model for antenatal care to improve communication. Int J Nurs Stud. 2016;54:84-94. https://doi.org/10.1016/j.ijnurstu.2015.03.018.

38. Jafari F, Eftekhar H, Fotouhi A, Mohammad K, Hantoushzadeh S. Comparison of maternal and neonatal outcomes of group versus individual prenatal care: a new experience in Iran. Health Care Women Int. 2012;31(7):571-84. https://doi.org/10.1080/07399331003646323.

39. Hofmeyr GJ, Hodnett ED. Antenatal care packages with reduced visits and perinatal mortality: a secondary analysis of the WHO antenatal care trial Comentary: routine antenatal visits for healthy pregnant women do make a difference. Reprod Health. 2013;10(1):20. https://doi.org/10.1186/1742-4755-10-20.

40. Lori JR, Ofosu-Darkwah H, Boyd CJ, Banerjee T, Adanu RMK, Banergee t, Adanu RM. Improving health literacy through group antenatal care: a prospective cohort study. BMC Pregnancy Childbirth2. 2017;17(1):228. https://doi.org/10.1186/s12884-017-1414-5.

41. Kabue MM, Grenier L, Suhowatsky S, et al. Group versus individual antenatal and first year postpartum care: Study protocol for a multi-country cluster randomized controlled trial in Kenya and Nigeria. Gates Open Res. 2019;2:56. https://doi.org/10.12688/gatesopenres.12867.2.

42. Sayinzoga F, Lundeen T, Gakwerere M, et al. Use of a facilitated group process to design and implement a group antenatal and postnatal care program in Rwanda. J Midwifery Womens Health. 2018;63(5):593-601. https://doi.org/10.1111/jmwh.12871.

43. Musange SF, Butrick E, Lundeen T, et al. Group antenatal care versus standard antenatal care and effect on mean gestational age at birth in Rwanda: protocol for a cluster randomized controlled trial. Gates Open Res. 2019;3:1548. https://doi.org/10.12688/gatesopenres.13053.1.

44. Mahmoud R, Ghani A. Perception toward conducting the the centering pregnancy model in the Egyptian teaching hospitals: a step to improve the quality of antenatal care. Eur J Biol Med Sci Res. 2014;2(2):45-54 https:// www.eajournals.org/journals/european-journal-of-biology-and-medicalscience-research-ejbmsr/vol-3issue1march-2015/perception-towardconducting-centering-pregnancy-model-egyptian-teaching-hospitals-stepimprove-quality-antenatal-care/. Accessed 20 Nov 2019.

45. Ruhl L, Christoffersen-Deb A, Elung'at J, Songok J. AfyaJamii: introducing a group prenatal and postnatal care model in Kenya. Ann Glob Health. 2015; 81(1):199. https://doi.org/10.1016/j.aogh.2015.02.957.
46. Penna LHG, Carinhanha Jl, Rodrigues RF. Consulta pre-natal colectiva: Una nueva propuesta para la atención integral. Rev Lat Am Enfermagem. 2008; 16(1):158-60. https://doi.org/10.1590/S0104-11692008000100024.

47. Arnold J, Morgan A, Morrison B. Paternal perceptions of and satisfaction with group prenatal care in Botswana. J Cult Competence Nurs Healthc. 2014;4(2):17-26. https://doi.org/10.9730/ojccnh.org/v4n2a2.

48. Sultana M, Mahumud RA, Ali N, et al. The effectiveness of introducing group prenatal care (GPC) in selected health facilities in a district of Bangladesh: study protocol. BMC Pregnancy Childbirth. 2017;17(1):48. https://doi.org/10. 1186/s12884-017-1227-6.

49. Lundeen T, Musange $\mathrm{S}$, Azman $\mathrm{H}$, et al. Nurses' and midwives' experiences of providing group antenatal and postnatal care at 18 health centers in Rwanda: a mixed methods study. PLoS One. 2019;14(7):e0219471. https:// doi.org/10.1371/journal.pone.0219471.

50. Musabyimana A, Lundeen T, Butrick E, et al. Before and after implementation of group antenatal care in Rwanda: a qualitative study of women's experiences. Reprod Health. 2019;16(1):90. https://doi.org/10.1186/ s12978-019-0750-5.

51. Group Care Global | Advancing Innovative Healthcare: Pregnancy and Beyond. http://groupcare.global/. Accessed 20 Nov 2019.

52. Noguchi L, Grenier L, Suhowatsky S, Oyetunji J, Wambua J, Smith J. Group antenatal care is associated with increased uptake of intermittent preventive treatment of malaria in pregnancy among women in Nigeria, compared to routine antenatal care: secondary analysis of a cluster randomized controlled trial. Am J Obstet Gynecol. 2018;219(6):640. https:// doi.org/10.1016/j.ajog.2018.10.080.

53. Grenier L, Suhowatsky S, Kabue MM, et al. Impact of group antenatal care (G-ANC) versus individual antenatal care (ANC) on quality of care, ANC attendance and facility-based delivery: a pragmatic cluster-randomized controlled trial in Kenya and Nigeria. PLoS One. 2019;14(10):e0222177. https://doi.org/10.1371/journal.pone.0222177.

54. Adaji SE, Jimoh A, Bawa U, et al. Women's experience with group prenatal care in a rural community in northern Nigeria. Int J Gynecol Obstet. 2019; 145(2):164-9. https://doi.org/10.1002/ijgo.12788.

55. Eluwa Gl, Adebajo SB, Torpey K, et al. The effects of centering pregnancy on maternal and fetal outcomes in northern Nigeria; a prospective cohort analysis. BMC Pregnancy Childbirth. 2018;18(1):158. https://doi.org/10.1186/ s12884-018-1805-2.

56. Chawanpaiboon S, Vogel JP, Moller AB, et al. Global, regional, and national estimates of levels of preterm birth in 2014: a systematic review and modelling analysis. Lancet Glob Health. 2019;7(1):e37-46. https://doi.org/10. 1016/S2214-109X(18)30451-0.

57. March of Dimes, PMNCH, Save the Children W. Born Too Soon: The Global Action Report on Preterm Birth. Geneva; 2012.

58. Curran GMGM, Bauer M, Mittman B, Pyne JMJM, Stetler C. Effectivenessimplementation hybrid designs: combining elements of clinical effectiveness and implementation research to enhance public health impact. Med Care. 2012;50(3):217-26. https://doi.org/10.1097/MLR. ob013e3182408812

59. Cromwell EA, Dow AE, Low D, et al. Barriers to successful early infant diagnosis of HIV infection at primary care level in Malawi. Pediatr Infect Dis J. 2015;34(3):273-5. https://doi.org/10.1097/INF.0000000000000625.

60. Bergh A-M, Arsalo I, Malan AF, Patrick M, Pattinson RC, Phillips N. Measuring implementation progress in kangaroo mother care. Acta Paediatr. 2005; 94(8):1102-8. https://doi.org/10.1080/08035250510028380.

61. Bergh A, Pattinson RC. Development of a conceptual tool for the implementation of kangaroo mother care; 2003. p. 709-14. https://doi.org/ 10.1080/08035250310002399.

62. Pattinson R, Arsalo I, Bergh A-M, Malan A, Patrick M, Phillips N. Implementation of kangaroo mother care: a randomized trial of two outreach strategies. Acta Paediatr. 2005;94(7):924-7. https://doi.org/10.1080/ 08035250510028399.

63. Smith $\mathrm{H}$, Asfaw AG, Aung KM, et al. Implementing the WHO integrated tool to assess quality of care for mothers, newborns and children: results and lessons learnt from five districts in Malawi. BMC Pregnancy Childbirth. 2017; 17(1):271. https://doi.org/10.1186/s12884-017-1461-y.

64. Wandersman A, Alia K, Cook BS, Hsu LL, Ramaswamy R. Evidence-based interventions are necessary but not sufficient for achieving outcomes in each setting in a complex world: empowerment evaluation, getting to outcomes, and demonstrating accountability. Am J Eval. 2016: 1098214016660613. https://doi.org/10.1177/1098214016660613. 
65. Wandersman A, Chien VH, Katz J. Toward an evidence-based system for innovation support for implementing innovations with quality: tools, training, technical assistance, and quality assurance/quality improvement. Am J Community Psychol. 2012;50(3-4):445-59. https://doi.org/10.1007/ s10464-012-9530-x.

66. Powell BJ, Waltz TJ, Chinman MJ, et al. A refined compilation of implementation strategies: results from the expert recommendations for implementing change (ERIC) project. Implement Sci. 2015;10(1):21. https:// doi.org/10.1186/s13012-015-0209-1.

67. WHO | Stillbirths. WHO. http://www.who.int/maternal_child_adolescent/ epidemiology/stillbirth/en/. Accessed 14 Dec 2017. Published. 2016.

68. Beusenberg M, Orley JH, World Health Organization. A User's Guide to the Self Reporting Questionnaire:; 1994.

69. Stewart RC, Umar E, Tomenson B, Creed F. Validation of screening tools for antenatal depression in Malawi-a comparison of the Edinburgh postnatal depression scale and self reporting questionnaire. J Affect Disord. 2013;150(3):1041-7. https://doi.org/10.1016/j.jad.2013.05.036 Accessed 10 Jan 2014.

70. Stewart RC, Kauye F, Umar E, et al. Validation of a Chichewa version of the Self-Reporting Questionnaire (SRQ) as a brief screening measure for maternal depressive disorder in Malawi, Africa. J Affect Disord. 2009;112(1â $€^{\prime \prime} 3$ ):126-34 http://www.sciencedirect.com/science/article/pii/S0165032 708001511.

71. Stewart RC, Bunn J, Vokhiwa M, et al. Common mental disorder and associated factors amongst women with young infants in rural Malawi. Soc Psychiatry Psychiatr Epidemiol. 2010;45(5):551-9. https://doi.org/10.1007/ s00127-009-0094-5.

72. Sanchis-Gomar F, Cortell-Ballester J, Pareja-Galeano H, Banfi G, Lippi G. Hemoglobin point-of-care testing. J Lab Autom. 2013;18(3):198-205. https:// doi.org/10.1177/2211068212457560.

73. Damschroder L, Hall C, Gillon L, et al. The consolidated framework for implementation research (CFIR): progress to date, tools and resources, and plans for the future. Implement Sci. 2015;10(S1):A12. https://doi.org/10.1186/ 1748-5908-10-S1-A12.

74. Maslach C, Jackson S, Leiter M. Maslach Burnout Inventory Manual. Third. Mountain View: CCP, Inc.; 1996.

75. Thorsen VC, Tharp ALT, Meguid T. High rates of burnout among maternal health staff at a referral hospital in Malawi: a cross-sectional study. BMC Nurs. 2011;10(1):9. https://doi.org/10.1186/1472-6955-10-9.

76. Khamisa N, Oldenburg B, Peltzer K, llic D. Work related stress, burnout, job satisfaction and general health of nurses. Int J Environ Res Public Heal Int Environ Res Public Heal. 2015;12:652-66. https://doi.org/10.3390/ ijerph120100652.

77. Mbindyo PM, Blaauw D, Gilson L, English M. Developing a tool to measure health worker motivation in district hospitals in Kenya. Hum Resour Health. 2009;7(1):40. https://doi.org/10.1186/1478-4491-7-40.

78. Mbindyo P, Gilson L, Blaauw D, English M. Contextual influences on health worker motivation in district hospitals in Kenya. Implement Sci. 2009;4(1):43. https://doi.org/10.1186/1748-5908-4-43.

79. CFIR Research team. Consolidated Framework for Implementation Research. http://www.cfirguide.org/. Accessed 15 Aug 2017.

80. Damschroder LJ, Aron DC, Keith RE, Kirsh SR, Alexander JA, Lowery JC. Fostering implementation of health services research findings into practice: a consolidated framework for advancing implementation science. Implement Sci. 2009;4(1):50. https://doi.org/10.1186/1748-5908-4-50.

81. Tufts University. ACASI, CASI and CAPI Software Systems: Easy Electronic Data Collection for your Research Studies. http://acasi.tufts.edu/home.htm. Accessed 1 Dec 2017.

82. Damschroder $L$, et al. Implementation and evaluation of the VA DPP clinical demonstration: protocol for a multi-site non-randomized hybrid effectiveness-implementation type III trial. Implement Sci. 2015;10:68.

83. Miles MB, Huberman AM, Saldaña J. Qualitative Data Analysis: A Methods Sourcebook. Thousand Oaks: SAGE Publications; 2013.

84. Stake RE. The Art of Case Study Research. Thousand Oaks: SAGE Publications; 1995.

85. Creswell JW, Clark VLP. Designing and Conducting Mixed Methods Research. Thousand Oaks: Sage Publications, Inc.; 2011.

86. Green C, a et al.Approaches to Mixed Methods Dissemination and Implementation Research: Methods, Strengths, Caveats, and Opportunities. Adm Policy Ment Health. 2014;42(5):508-23. https://doi.org/10.1007/s10488014-0552-6.
87. Scaccia JP, Cook BS, Lamont A, et al. A practical implementation science heuristic for organizational readiness: $R=$ MC2. J Community Psychol. 2015: 43(4):484-501. https://doi.org/10.1002/jcop.21698.

88. Glasgow RE, Vinson C, Chambers D, Khoury MJ, Kaplan RM, Hunter C. National Institutes of Health approaches to dissemination and implementation science: current and future directions. Am J Public Health. 2012;102(7):1274-81. https://doi.org/10.2105/AJPH.2012.300755.

89. National Statistical Office (NSO) [Malawi] and ICF. Malawi Demographic and Health Survey 2015-16. Zomba and Rockville: NSO and ICF; 2017.

\section{Publisher's Note}

Springer Nature remains neutral with regard to jurisdictional claims in published maps and institutional affiliations.

\section{Ready to submit your research? Choose BMC and benefit from:}

- fast, convenient online submission

- thorough peer review by experienced researchers in your field

- rapid publication on acceptance

- support for research data, including large and complex data types

- gold Open Access which fosters wider collaboration and increased citations

- maximum visibility for your research: over $100 \mathrm{M}$ website views per year

At BMC, research is always in progress.

Learn more biomedcentral.com/submissions 